\title{
Comments on Whether or Not Traditional Chinese Medicine and Acupuncture are Pseudosciences
}

\author{
Tong zheng Hong* \\ Department of Pharmacology, Taiwan \\ *Corresponding author: Tong zheng Hong, Department of Pharmacology, Taiwan.
}

\begin{abstract}
Whether or not the traditional Chinese medicine (TCM) and acupuncture are science remains controversial. It seems that both should not be judged with the criteria based on the deductive reasoning because it goes beyond doubt that the different logical reasoning models between the East and the West need more conversation. Issues affecting the efficacy of TCM and acupuncture like Collection, Processing, Acupuncture as part of the health system, Designing Pattern-based research, Locating acupoints accurately, and Proper prescriptions are presented for discussion for the modernization and the scientization of TCM and acupuncture.
\end{abstract}

Keywords: Inductive/Deductive Reasoning; Acupoint; Yin-Yang; Pattern Identification

\section{Introduction}

Acupuncture and the traditional Chinese Medicine (TCM) are complex, developed with the philosophical principles and reflecting Chinese thought, which is used to place the human body into a large system through the observation of nature and has been recognized and practiced totreat various diseases and symptoms worldwide. It's been argued academically and clinically for long whether or not the TCM and acupuncture should be treated as science or pseudoscience. The Chinese are used to the inductive reasoning for more than five thousand years, compared to deductive reasoning of in the West. Based on this reasoning model, the TCM and acupuncture features similarities with Yin-Yang, Qi-Blood, the Five Elements, and Pattern identification in theories. In addition to the scientific evidence with modern techniques, such as FMRI and CT, to examine the effects of the TCM and acupuncture, it seems to suggest that some key issues deserve attention. Having the better understanding and learning of the TCM and acupuncture, which will greatly promote research in the TCM and acupuncture, may be beneficial to modernization and scientization of the TCM and acupuncture and rethinking the definition of science.

\section{Current Issues}

\section{Collection}

The clinical use of Chinese herbs must follow the TCM theories and be based on the patient's specific conditions with accurate diagnosis, following the principles of pattern identification. Ban-xia (Pinellia ternate) is effective to drain phlegm caused by dampness. Ban literally in Chinese means in the middle summer of which indicates the best harvest time is in the mid-summer between May 6 (lixia,) when summer begins and June 21, maximum summer (xiazhi) for the maximum constituents and best quality. However, this rule seems to be difficult to follow, suggesting why the efficacy is as good as expected for the clinical outcomes [1].

\section{Processing}

In order to facilitate the use of Chinese herbs to meet the clinical needs, herb processing is required because it can generally reduce toxicity, alter energetic nature, and reinforce efficacy to increase the therapeutic effectiveness. Radix Bupleuri (RB) bearing the properties of spicy, cool, and bitter is one of the 
most popular traditional Chinese medical herbs to treat Liverrelated diseases. Radix Bupleuri is derived from the dried roots of Bupleurum scorzonerifolium Willd (Nan Chaihu) and Bupleurum chinense DC. (Pei Chaihu), which is the main ingredient of the most frequently used preparations Da Chai Hu decoction and Xiao Chai $\mathrm{Hu}$ decoction in the Treatise on Injury by Cold (Shang Han Lun). The major differences between Bupleurum scorzonerifolium and Willd Bupleurum chinense DC. are the indication of Bupleurum chinense DC. is to disperse stagnated Liver qi while Bupleurum scorzonerifolium Willd is used to reinforce Yang Qi in the pattern of Middle Jiao deficiency [1]. However, whether or not Bei Chaihu (Bupleurum chinense DC.) is selected as the components of these two formulas remains uncertain since it is not specified by the author Zhang Zhongjing of Treatise on Injury by Cold (Shang Han Lun), even though Bupleurum chinense DC. is mostly selected for clinical use. On the other hand, the evidence-based researcher shows the levels of neurotransmitters in the frontal cortex and hippocampus can be regulated significantly with the use of vinegarbaked Bupleuri Radix In addition, vinegar-baked Bupleuri Radix can be more effective for relieving the depressed Liver qi and bring about the better antidepressant effect than unprocessed Bupleuri Radix $[1,2]$.

\section{Acupuncture as Part of The Health System}

Whether or not acupuncture is science remains controversial only because this issue is presented and discussed by those who are used to the deductive reasoning. However, the objective evidence of the population of China has given the answer, explaining the statuses of TCM and acupuncture in China and the Chinese communities around the world. TCM and acupuncture had been the major approaches in the health system in the ancient China until the Qing dynasty. In the first half of the nineteen century, the population of China accounted for one-third of the world population even though China had been tortured by the twenty one major wars and battles, leading to the fluctuation of population. It is estimated the population was about forty two million in the Qin dynasty (221206 BC) and increased to eighty six million in the Han dynasty (206 BC- $220 \mathrm{AD}$ ). The population moved around 100 million to 150 million until 1700s after the Ming dynasty (1368-1344) and earlier period of the Qing dynasty (1644-1912). More impressive evidence is that the population doubled in a century in the period between 1749 and $1851[3,4]$.

\section{Designing Pattern-Based Research}

It is noted that most of the contemporary scientific researches examining the efficacy of acupuncture and TCM only focuses on diseases, instead of following the pattern identification. It is understood that a disease refers to the loss of the balance of YinYang [3]. TCM and acupuncture see the body as a miniature of the universe in diagnosis and treatment and believe the body acts with the universe, which is totally different from the Western medicine. Yin and Yang are the philosophical concepts and abstract with the functions broader than the anatomical knowledge of the Western medicine. On the other hand, TCM emphasizes the congenital constitution passed from the parents, which is equivalent to the concept of gene centered in the Precision medicine [5]. It is agreed that criteria for evaluating Pizza should not be used to assess Burrito. In other words, the research designs not following the pattern identification cannot accurately exemplify the TCM and acupuncture theories to a great extent because they are breaking the rules and principles developed with the inductive reasoning model [6].

\section{Locating Acupoints Accurately}

How to accurately locate an acupoint is the key to the successful treatment. For, example, LIV1 (Da Dun) is the most commonly used acupoint in the acupuncture treatment for regulating Qi in the lower jiao and Liver qi to alleviate pain. Unfortunately, whether this acupoint is located on the dorsal aspect of the big toe, at the junction of lines drawn along the lateral border of the nail and the base of the nail, approximately 0.1 cun from the corner of the nail [7] or on the lateral side of the terminal phalanx of the great toe, 0.1 cun from the corner of the nail [8] remains controversial in the clinical practice and textbooks.

\section{Proper Prescription}

As discussed above, Pattern identification is vital for the successful treatment outcomes. The historical developments show that an acupuncturist may face the following challenging issues, such as only selecting traditional acupoints for use, only selecting extra acupoints, or the combinations of traditional acupoints with extra acupoints, while a TCM practitioner needs to consider the processed/unprocessed herbs, the collection way of the herbs. Up to now, there has not scientific evidence to show which choice can reach the best treatment outcomes. In other words, these issues depend on acupuncturists' wisdom and experience.

\section{Discussion}

There has not been sufficient scientific evidence to prove the meridians exist. However, acupuncture is recommended by the WHO [8] and widely known for the effectiveness in the treatment of pain. Current development and the acceptance of acupuncture in the West may suggest whether or not the definition of science can apply to the acupuncture and the TCM still deserves attention and discussion. The efficacy in reducing pain is one of the main reasons why it has been recognized to be the effective approach around the world. The plentiful evidence on the effects of acupuncture on specific painful conditions has been presented. The systematic review of 13 trials of acute pain found that acupuncture is more effective than both sham needling and painkillers [9-11]. Banxia (Pinellia ternate) is used to drain phlegm effectively caused by dampness, which suggests that it should not be selected to fight against phlegm caused by dryness. This example highlights the importance of pattern identification for the optimal and best 
outcomes. In addition, the nature of the Chinese herbs is changed when it is processed, which indicates how to identify accurately the characteristics of Chinese herbs is crucial to the clinical use. The historical developments show that there are challenges ahead of the TCM and acupuncture learners and practitioners, such as whether it is effective to select the unprocessed herbs, research design following the pattern identification, only use acupoints on the traditional regular meridians, when to combine the regular acupoints with the extra acupoints, how to decide the accurate needling duration, etc.

\section{Conclusion}

The expected treatment and experiment outcomes will not occur until the acupuncture and TCM patterns are followed and taken into the consideration since there exist some fundamental differences between the Western medicine and the TCM, including acupuncture. On the other hand, it deserves attention that whether the definition of science developed with deductive reasoning can apply to the Yin-Yang and the Five Elements theories derived from the inductive reasoning needs more discussion.

\section{References}

1. Hong TZ (2018) Challenges in Learning and Understanding Traditional Chinese Medicine and Acupuncture. Open Acc J Comp \& Alt Med.
2. Lei $\mathrm{T}$, Chen S, Wang K, Zhang D, Dong L, et al. (2017) Characterization and discrimination of raw and vinegar-baked Bupleuri Radix based on UHPLC-Q-TOF-MS coupled with multivariate statistical analysis. Biomedical Chromatography 32(2).

3. List of Chinese wars and battles. Wikipedia.

4. Demographics of China. Wikipedia.

5. Hong TZ (2018) Considerations of Acupuncture Protocol for Treating Heart Blood Deficiency-Patterned Heart Diseases. Open Acc J Comp \& Alt Med 1(2).

6. Hong TZ (2018) A Review for Integrating Western and Chinese Medicines in Primary Dysmenorrhea. Advancements Bioequiv Availab 2(1): 1-4.

7. Hong TZ (2018) Challenges in Learning and Understanding Traditional Chinese Medicine and Acupuncture. Open Acc J Comp \& Alt Med 1(1): 5-9.

8. Deadman, Peter, Al-Khadjafi, Mazun, Baker, et al. (2007) A Manual of Acupuncture.

9. Cheng X, Deng L (1987) Chinese acupuncture and moxibustion.

10. Russell D, Koppelman KH. Acupuncture For Pain. Evidenc Based Acupuncture.

11. Xiang A, Cheng K, Shen X, Xu P, Liu S (2017) The immediate analgesic effect of acupuncture for pain: A systematic review and meta-analysis. Evidence-based Complementary and Alternative Medicine 2017: 1-13.

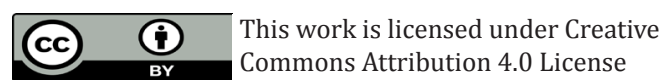

To Submit Your Article Click Here:

Submit Article

DOI: $10.32474 /$ LOJPCR.2019.01.000111

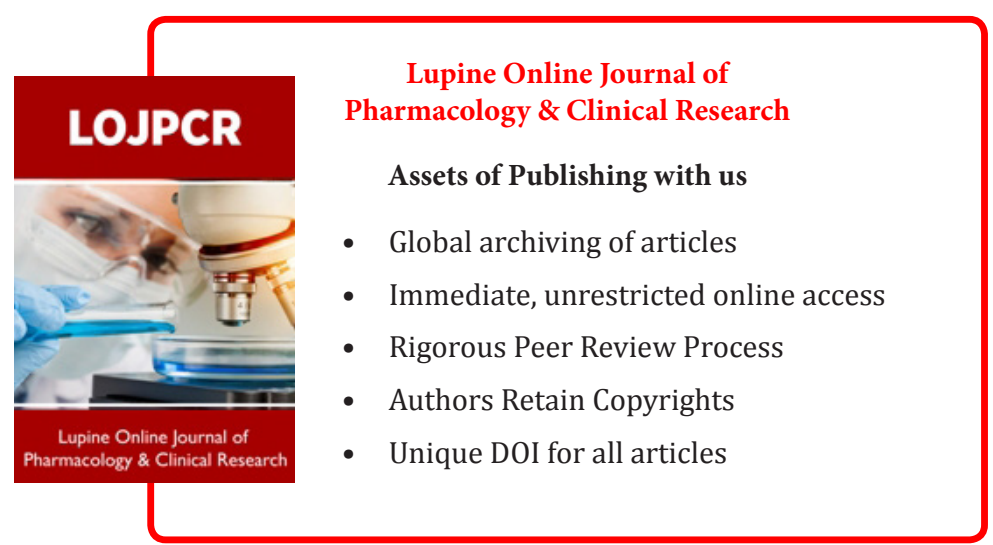

